(c) (1)

Supplement of

\title{
Body weight prediction using body size measurements in Fleckvieh, Holstein, and Brown Swiss dairy cows in lactation and dry periods
}

Leonhard Gruber et al.

Correspondence to: Maria Ledinek (ledinekmaria@yahoo.de)

The copyright of individual parts of the supplement might differ from the CC BY 4.0 License. 
Table S1. Description of the estimation and validation subset for Model $_{\mathrm{HG}}$ BG during lactation $(\mathrm{HG}=$ heart girth; $\mathrm{BG}=$ belly girth $)$.

\begin{tabular}{|c|c|c|c|c|c|c|c|c|c|c|c|c|}
\hline \multirow[t]{2}{*}{ Trait } & \multicolumn{6}{|c|}{ Estimation subset } & \multicolumn{6}{|c|}{ Validation subset } \\
\hline & $n$ & Mean & $\begin{array}{l}\text { Standard } \\
\text { deviation }\end{array}$ & $\begin{array}{c}\text { Variation } \\
\text { coefficient }\end{array}$ & Minimum & Maximum & $n$ & Mean & $\begin{array}{l}\text { Standard } \\
\text { deviation }\end{array}$ & $\begin{array}{c}\text { Variation } \\
\text { coefficient }\end{array}$ & $\begin{array}{c}\text { Minimu } \\
\mathrm{m}\end{array}$ & Maximum \\
\hline Body weight, kg & 32,116 & 699 & 89.7 & 12.8 & 400 & 1,088 & 8,013 & 699 & 89.6 & 12.8 & 403 & 1,042 \\
\hline Body weight estimated, $\mathrm{kg}$ & & & & & & & 8,013 & 699 & 80.6 & 11.5 & 422 & 1,011 \\
\hline Heart girth, $\mathrm{cm}$ & 32,116 & 210 & 10.4 & 4.9 & 166 & 257 & 8,013 & 210 & 10.3 & 4.9 & 168 & 253 \\
\hline Belly girth, cm & 32,116 & 255 & 13.9 & 5.5 & 193 & 302 & 8,013 & 255 & 13.9 & 5.5 & 204 & 312 \\
\hline Stature, $\mathrm{cm}$ & 8,439 & 146 & 4.5 & 3.1 & 128 & 163 & 2,122 & 146 & 4.7 & 3.2 & 130 & 163 \\
\hline Body length, cm & 7,938 & 90 & 5.6 & 6.2 & 73 & 110 & 1,984 & 91 & 5.7 & 6.3 & 74 & 111 \\
\hline Pelvis length, $\mathrm{cm}$ & 8,436 & 56 & 3.0 & 5.3 & 46 & 68 & 2,121 & 56 & 3.0 & 5.4 & 45 & 68 \\
\hline Body depth, cm & 8,437 & 84 & 4.5 & 5.4 & 67 & 99 & 2,119 & 84 & 4.5 & 5.4 & 70 & 99 \\
\hline Hip width, cm & 8,435 & 57 & 3.4 & 5.9 & 45 & 68 & 2,119 & 57 & 3.4 & 6.0 & 46 & 67 \\
\hline Pin width, $\mathrm{cm}$ & 8,425 & 39 & 4.8 & 12.2 & 27 & 59 & 2,122 & 39 & 4.6 & 11.8 & 28 & 58 \\
\hline Knee width, cm & 8,415 & 54 & 5.5 & 10.3 & 34 & 71 & 2,124 & 53 & 5.5 & 10.2 & 36 & 69 \\
\hline BCS, points $1-5$ & 31,944 & 3.16 & 0.59 & 18.7 & 1.00 & 5.00 & 7,971 & 3.16 & 0.59 & 18.7 & 1.00 & 5.00 \\
\hline Muscle score, points $1-10$ & 31,919 & 5.1 & 1.5 & 29.8 & 1.0 & 9.0 & 7,968 & 5.1 & 1.5 & 29.6 & 1.0 & 9.0 \\
\hline Parity & 32,116 & 3.0 & 2.0 & 67.2 & 1 & 14 & 8,013 & 3.0 & 2.0 & 67.3 & 1 & 13 \\
\hline Day relative to calving & 32,116 & 159 & 96 & 60 & 1 & 364 & 8,013 & 158 & 97 & 61 & 1 & 364 \\
\hline
\end{tabular}




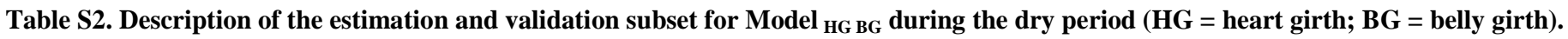

\begin{tabular}{|c|c|c|c|c|c|c|c|c|c|c|c|c|}
\hline \multirow[t]{2}{*}{ Trait } & \multicolumn{6}{|c|}{ Estimation subset } & \multicolumn{6}{|c|}{ Validation subset } \\
\hline & $n$ & Mean & $\begin{array}{l}\text { Standard } \\
\text { deviation }\end{array}$ & $\begin{array}{c}\text { Variation } \\
\text { coefficient }\end{array}$ & Minimum & Maximum & $n$ & Mean & $\begin{array}{l}\text { Standard } \\
\text { deviation }\end{array}$ & $\begin{array}{c}\text { Variation } \\
\text { coefficient }\end{array}$ & $\begin{array}{c}\text { Minimu } \\
\mathrm{m}\end{array}$ & Maximum \\
\hline Body weight, kg & 3,440 & 794 & 92.1 & 11.6 & 506 & 1,105 & 872 & 794 & 92.3 & 11.6 & 534 & 1,108 \\
\hline Body weight estimated, $\mathrm{kg}$ & & & & & & & 872 & 796 & 81.5 & 10.2 & 586 & 1,085 \\
\hline Heart girth, $\mathrm{cm}$ & 3,440 & 218 & 11.0 & 5.1 & 182 & 264 & 872 & 218 & 10.9 & 5.0 & 188 & 258 \\
\hline Belly girth, cm & 3,440 & 271 & 13.2 & 4.9 & 227 & 320 & 872 & 271 & 13.6 & 5.0 & 231 & 314 \\
\hline Stature, $\mathrm{cm}$ & 909 & 146 & 4.7 & 3.2 & 131 & 163 & 264 & 145 & 4.4 & 3.0 & 130 & 156 \\
\hline Body length, cm & 846 & 91 & 5.9 & 6.4 & 75 & 109 & 246 & 92 & 5.4 & 5.8 & 75 & 105 \\
\hline Pelvis length, $\mathrm{cm}$ & 909 & 56 & 3.1 & 5.4 & 48 & 66 & 264 & 56 & 3.1 & 5.5 & 45 & 66 \\
\hline Body depth, cm & 908 & 86 & 4.2 & 4.9 & 71 & 99 & 264 & 86 & 4.3 & 5.0 & 74 & 99 \\
\hline Hip width, cm & 909 & 58 & 3.4 & 5.8 & 47 & 68 & 262 & 58 & 3.3 & 5.8 & 48 & 70 \\
\hline Pin width, $\mathrm{cm}$ & 907 & 40 & 4.9 & 12.4 & 28 & 57 & 261 & 40 & 4.7 & 11.9 & 27 & 56 \\
\hline Knee width, cm & 907 & 55 & 6.0 & 10.8 & 36 & 70 & 260 & 55 & 6.5 & 11.8 & 38 & 71 \\
\hline BCS, points $1-5$ & 3,429 & 3.61 & 0.57 & 15.9 & 1.50 & 5.00 & 871 & 3.62 & 0.58 & 15.9 & 1.25 & 5.00 \\
\hline Muscle score, points $1-10$ & 3,423 & 5.9 & 1.5 & 25.1 & 1.0 & 9.0 & 867 & 5.9 & 1.5 & 25.4 & 2.0 & 9.0 \\
\hline Parity & 3,440 & 2.8 & 1.8 & 65.4 & 1 & 13 & 872 & 2.8 & 1.9 & 67.3 & 1 & 12 \\
\hline Day relative to calving & 3,440 & -24 & 15 & -61 & -56 & -1 & 872 & -25 & 15 & -58 & -56 & -1 \\
\hline
\end{tabular}


Table S3. Number of data records of the body weight prediction models Model $_{\mathrm{HG}}$ BG and Model $_{\mathrm{HG}} \mathrm{BG}$ HW separated for lactation and dry period (fixed effect genotype and parity).

\begin{tabular}{|c|c|c|c|c|c|c|c|c|c|c|c|}
\hline \multirow[t]{2}{*}{ DRY-LAC } & \multirow[t]{2}{*}{ EST-VAL $^{2}$} & \multirow[t]{2}{*}{$n$} & \multicolumn{5}{|c|}{ Genotype $^{3}$} & \multicolumn{4}{|c|}{ Parity } \\
\hline & & & FV & FV $\times$ RH_m & FV $\times$ RH_h & $\mathrm{HF}$ & BS & 1 & 2 & $3+4$ & $\geq 5$ \\
\hline \multicolumn{12}{|c|}{ Model including the body measurements heart girth and belly girth (Model ${ }_{\mathrm{HG}} \mathrm{BG}$ ) } \\
\hline LAC & EST & 32,116 & 13,457 & 3,860 & 1,872 & 5,171 & 7,756 & 9,136 & 7,100 & 9,120 & 6,760 \\
\hline LAC & VAL & 8,013 & 3,330 & 988 & 478 & 1,300 & 1,917 & 2,290 & 1,754 & 2,288 & 1,681 \\
\hline DRY & EST & 3,440 & 1,506 & 423 & 207 & 502 & 802 & 1009 & 848 & 1007 & 576 \\
\hline DRY & VAL & 872 & 402 & 93 & 43 & 121 & 213 & 267 & 201 & 250 & 154 \\
\hline \multicolumn{12}{|c|}{ Model including the body measurements heart girth, belly girth, and hip width (Model ${ }_{\mathrm{HG} \mathrm{BG} \mathrm{HW}}$ ) } \\
\hline LAC & EST & 8,474 & 3,530 & 1,090 & 495 & 1,403 & 1,956 & 2,292 & 1,898 & 2,503 & 1,781 \\
\hline LAC & VAL & 2,080 & 867 & 262 & 119 & 351 & 481 & 564 & 487 & 587 & 442 \\
\hline DRY & EST & 909 & 370 & 99 & 46 & 158 & 236 & 274 & 236 & 239 & 160 \\
\hline DRY & VAL & 262 & 104 & 33 & 22 & 36 & 67 & 60 & 79 & 72 & 51 \\
\hline
\end{tabular}

${ }^{1} \mathrm{DRY}=$ dry period; $\mathrm{LAC}=$ lactation

${ }^{2} \mathrm{EST}=$ data set for estimation; $\mathrm{VAL}=$ data set for validation

${ }^{3} \mathrm{FV}=$ Fleckvieh $($ Red Holstein genes $\leq 10.0 \%) ; \mathrm{RH}=$ Red Holstein; HF = Holstein Friesian; $\mathrm{m}=$ medium proportion of RH genes $(>10.0-\leq 44.5 \%) ; \mathrm{h}=$ high proportion of RH genes (> $44.5 \%)$; BS = Brown Swiss 
Table S4. Number of data records of the body weight prediction models Model ${ }_{\mathrm{HG}}$ BG and Model $_{\mathrm{HG}} \mathrm{BG}$ HW separated for lactation and dry period (fixed effect of physiological stage: lactation and dry period).

\begin{tabular}{|c|c|c|c|c|c|c|c|c|c|c|c|c|c|c|c|c|c|c|}
\hline \multirow{2}{*}{$\begin{array}{l}\text { DRY- } \\
\text { LAC }^{1}\end{array}$} & \multirow{2}{*}{$\begin{array}{l}\text { EST- } \\
\text { VAL }^{2} \\
\end{array}$} & \multicolumn{8}{|c|}{ Dry period (weeks ante partum) } & \multicolumn{9}{|c|}{ Lactation (months post partum, 28 days per month) } \\
\hline & & $7-8$ & $5-6$ & $3-4$ & $1-2$ & 1 & 2 & 3 & 4 & 5 & 6 & 7 & 8 & 9 & 10 & 11 & 12 & 13 \\
\hline \multicolumn{19}{|c|}{ Model including the body measurements heart girth and belly girth (Model ${ }_{\mathrm{HG}} \mathrm{BG}$ ) } \\
\hline LAC & EST & & & & & 2,950 & 3,054 & 2,944 & 2,909 & 2,888 & 2,695 & 2,765 & 2,618 & 2,644 & 2,587 & 2,020 & 1,284 & 758 \\
\hline LAC & VAL & & & & & 791 & 777 & 690 & 730 & 659 & 732 & 715 & 670 & 644 & 543 & 493 & 346 & 223 \\
\hline DRY & EST & 483 & 860 & 1,028 & 1,069 & & & & & & & & & & & & & \\
\hline DRY & VAL & 131 & 237 & 272 & 232 & & & & & & & & & & & & & \\
\hline \multicolumn{19}{|c|}{ Model including the body measurements heart girth, belly girth, and hip width (Model ${ }_{\mathrm{HG} \mathrm{BG} \mathrm{HW}}$ ) } \\
\hline LAC & EST & & & & & 685 & 844 & 773 & 828 & 760 & 726 & 739 & 662 & 685 & 635 & 553 & 367 & 217 \\
\hline LAC & VAL & & & & & 153 & 225 & 185 & 169 & 180 & 180 & 177 & 170 & 158 & 174 & 149 & 109 & 51 \\
\hline DRY & EST & 131 & 273 & 290 & 215 & & & & & & & & & & & & & \\
\hline DRY & VAL & 46 & 75 & 94 & 47 & & & & & & & & & & & & & \\
\hline
\end{tabular}

